\title{
Design of controlled release PLGA microspheres for hydrophobic fenretinide
}

Ying Zhang ${ }^{1,2}$, Christian Wischke ${ }^{1,3}$, Sachin $\mathrm{Mittal}^{4}$, Amitava Mitra ${ }^{5}$ and Steven P. Schwendeman $1, *$

1 Department of Pharmaceutical Sciences, University of Michigan, 428 Church Street, Ann Arbor, Michigan 48109, USA

2 Current address: Critical \& Chronic Care Solutions Division, 3M, St Paul, MN, 55144

3 Current addresses: Institute of Biomaterial Science and Berlin-Brandenburg Center for Regenerative Therapies, Helmholtz-Zentrum Geesthacht, Kantstr. 55, 14513 Teltow, Germany.

4 Pharmaceutical Sciences and Clinical Supply, Merck Research Laboratories (MRL), Merck \& Co., Kenilworth, NJ, 07033, USA

5 Pharmaceutical Sciences and Clinical Supply, Merck Research Laboratories (MRL), Merck \& Co., West Point, PA, 19486, USA

* Corresponding author. Tel: +1-734-615-6574; Fax: + 1-734-615-6162

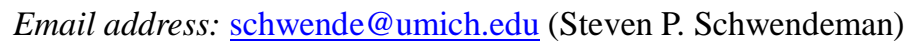




\section{Supporting information}

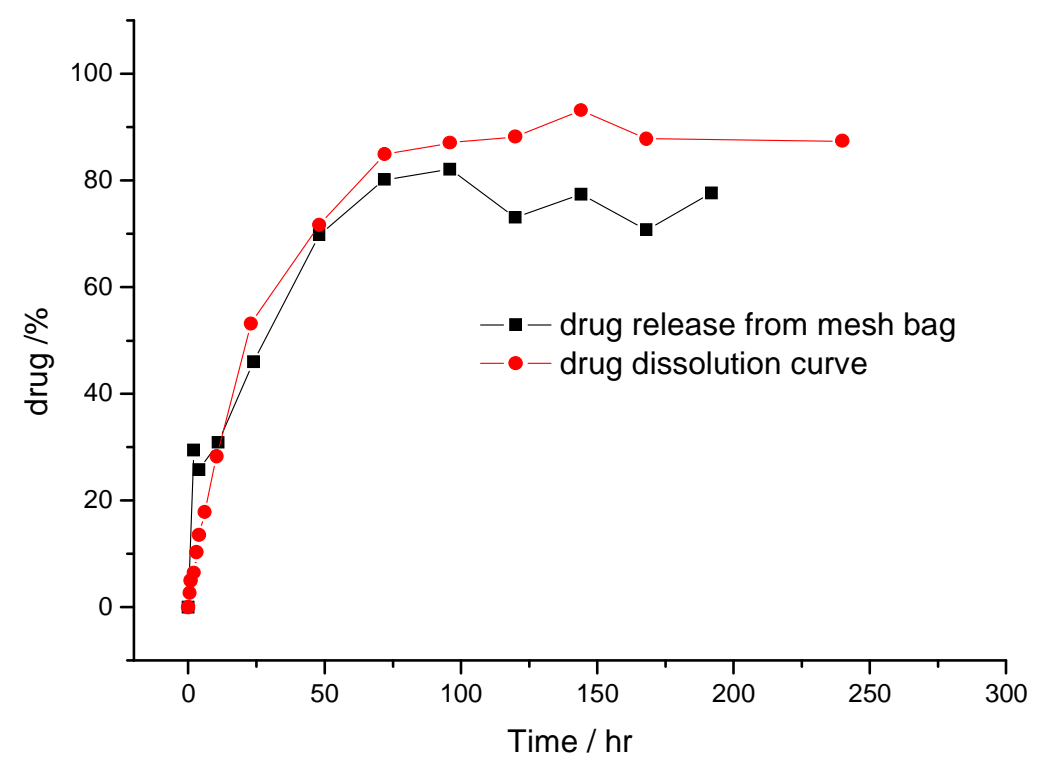

Figure S1. Comparison of pure fenretinide release from mesh bag and dissolution in $\operatorname{PBST}(0.1 \%$ Tween 20$)$

The release of pure drug was conducted as follows: 1-2 mg of pure fenretinide particles were placed in the mesh bags, sealed by an impulse heat sealer (Model: AIE-210C, American International Electric Inc, Whittier, CA). Mesh bags were placed in $1 \mathrm{~L} \mathrm{PBST}$ at $37^{\circ} \mathrm{C}$ with constant stirring. At predetermined time intervals, the supernatant was analyzed by HPLC, drug release percent was determined by the concentration of the drug in the supernatant. The remaining fenretinide in the mesh bags was lyophilized and analyzed by HPLC to check the mass balance. The dissolution study was conducted as follows: $1.70 \mathrm{mg}$ of fenretinide particles were placed in $1 \mathrm{~L}$ PBST $(0.1 \%$ Tween 20$)$ at $37^{\circ} \mathrm{C}$ with continuous stirring; at predetermined time intervals, $1 \mathrm{ml}$ PBST was removed, centrifugated, filtered and analyzed by HPLC. $1 \mathrm{ml}$ fresh PBST was added to maintain 1L volume). Please note that the mass balance did not reach up to $100 \%$ due to the instability of fenretinide in PBST at $37^{\circ} \mathrm{C}$. This issue could be overcome by frequently replacing entire release 
media and measure the remaining drug in the microspheres.
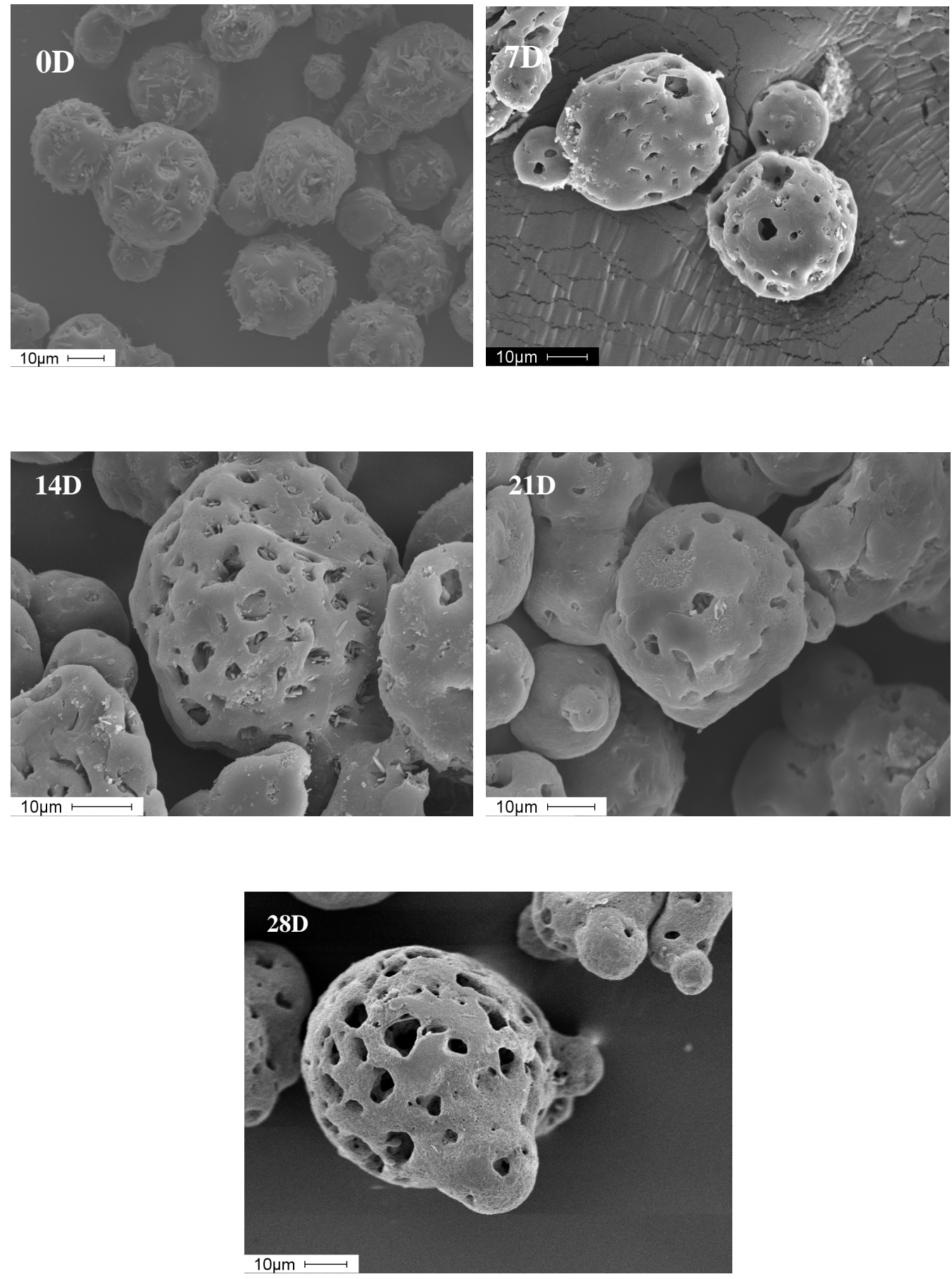

Figure S2. SEMs of microspheres (Formulation 3) incubation in PBST at $37^{\circ} \mathrm{C}$ over time. 